\title{
MEROMORPHIC PRODUCTS DETERMINING NEAR-FIELDS
}

\author{
PETER FUCHS and C. J. MAXSON
}

(Received 13 August 1987)

Communicated by B. J. Gardner

\begin{abstract}
In this paper we continue our investigations of a construction method for subnear-rings of $M(G)$ proposed by $\mathrm{H}$. Wielandt. For a meromorphic product $H, H \subseteq G^{k}, G$ finite, we obtain necessary and sufficient conditions for $M(G, k, H)$ to be a near-field.

1980 Mathematics subject classification (Amer. Math. Soc.): primary 12 K 05, 16 A 76; secondary $20 \mathrm{E} 10$.
\end{abstract}

\section{Introduction}

Let $G$ be a group written additively and $k$ a positive integer, $k \geq 2$. R. Remak has pointed out in [4] and [5] that one can construct subgroups of the direct power $G^{k}$ as follows. For $j \in\{1,2, \ldots, k\}$, let $B_{j}$ be a subgroup of $G, B_{j}$ a normal subgroup of $B_{j}$ such that $B_{j} / B_{j} \cong B_{j+1} / \bar{B}_{j+1}$ with isomorphisms $\sigma_{j}, j \in\{1, \ldots, k-1\}$. Let $\alpha$ be an ordinal, $\left\{b_{i \eta} \mid \eta<\alpha\right\}$ a set of coset representatives of $B_{1}$ in $B_{1}$ where $b_{10}=0$ and define a subset $H \subseteq G^{k}$ by

$$
H=\bigcup_{\eta<\alpha}\left[\left(b_{1 \eta}+B_{1}\right) \times \prod_{j=1}^{k-1}\left(\sigma_{j} \circ \sigma_{j-1} \circ \cdots \circ \sigma_{1}\left(b_{1 \eta}+B_{1}\right)\right)\right] .
$$

Then $H$ is called a $k$-fold meromorphic product and will be denoted by $H=B_{1} / B_{1} \underset{\sigma_{1}}{\times} B_{2} / B_{2} \underset{\sim}{\times} \cdots{\underset{\sim}{\sigma_{k-1}}}_{\sigma_{k}} B_{k} / B_{k}$. It is straightforward to verify that $H$ is a subgroup of $G^{k}$. However, only for $k=2$ can every subgroup of $G^{k}$ be obtained as a meromorphic product. Let $M(G)=\{f: G \rightarrow G\}$ act

(C) 1989 Australian Mathematical Society 0263-6115/89 \$A2.00+0.00 
on $G^{k}$ componentwise. For any subgroup $H$ of $G^{k}$ we define $M(G, k, H)=$ $\{f \in M(G) \mid f(H) \subseteq H\}$. These $M(G, k, H)$ are subnear-rings of $M(G)$ with identity id: $G \rightarrow G, \operatorname{id}(x)=x$, for all $x \in G$.

For $k=2$ it was shown in [1] that whenever $M(G, 2, H)$ is a near-field then it must be a field and $H$ is of the form $G /\{0\} \times G /\{0\}$. This result does not hold for $k \geq 3$. However, in this paper we show that every finite near-field arises from a meromorphic product of the form $B_{1} /\{0\} \times \cdots \times B_{k} /\{0\}$. More generally, for an arbitrary meromorphic product $H$, we obtain necessary and sufficient conditions for $M(G, k, H)$ to be a near-field. For a subset $S$ of $G$ we let $S^{*}=S \backslash\{0\}$.

\section{Characterization results}

We first show that any finite near-field arises from a meromorphic product.

THEOREM 2.1. Let $N$ be a zero-symmetric finite near-field. Then there exists a group $G$, a positive integer $k$ and a subgroup $H$ of $G^{k}$ where

$$
H=B_{1} /\{0\} \underset{\sim}{\times_{\sigma_{1}}} B_{2} /\{0\} \underset{\sim}{\underset{\sigma_{2}}{\times}} \cdots{\underset{\sim}{\sigma_{k-1}}}_{\boldsymbol{x}_{k}} B_{k} /\{0\}
$$

such that $N=M(G, k, H)$.

Proof. Let $G$ be a finite group such that $N$ is a subnear-field of $M(G)$. If $G^{*}=\left\{x_{1}, x_{2}, \ldots, x_{k}\right\}$ then we know $N x_{i} \cong N x_{j}$ as $N$-subgroups via $\sigma_{i j}: n x_{i} \mapsto n x_{j}, i, j \in\{1,2, \ldots, k\}$. Let

$$
H=N x_{1} \underset{\sim}{\times} N x_{\sigma_{12}} \underset{\sim}{\underset{\sigma_{23}}{x}} \cdots \underset{\sim}{\underset{\sigma_{k-1 k}}{x}} N x_{k} .
$$

Clearly $N \subseteq M(G, k, H)$. On the other hand, for $\left(x_{1}, x_{2}, \ldots, x_{k}\right) \in H$ and $m \in M(G, k, H), m\left(x_{1}, \ldots, x_{k}\right)=\left(m\left(x_{1}\right), \ldots, m\left(x_{k}\right)\right) \in H$. Now $m\left(x_{1}\right) \in$ $N x_{1}$ so $m\left(x_{1}\right)=f\left(x_{1}\right)$ for some $f \in N$. But the only $k$-tuple in $H$ with $f\left(x_{1}\right)$ as first component is $\left(f\left(x_{1}\right), f\left(x_{2}\right), \ldots, f\left(x_{k}\right)\right)$. Hence $f\left(x_{i}\right)=m\left(x_{i}\right)$ for all $x_{i} \in G^{*}$ and so $m=f \in N$.

We have shown that every finite near-field can be represented using a meromorphic product without quotients, that is, by using a meromorphic product of the form $B_{1} /\{0\} \times \cdots \times B_{k} /\{0\}$. Conversely one would like to characterize those meromorphic products without question that determine near-fields. In fact we consider the more general situation of meromorphic products with quotients, $H=B_{1} / B_{1} \times \cdots \times B_{k} / B_{k}$ and determine, in terms of properties of $H$, when $M(G, k, H)$ is a near-field. The "without quotients" case then follows as a corollary. 
Throughout this section all structures are finite. We first fix some notation and give some definitions. Let $H=B_{1} / \bar{B}_{1} \underset{\sim}{\underset{\sigma_{1}}{x}} \cdots \underset{\sim}{\underset{\sigma_{k-1}}{\times}} B_{k} / \bar{B}_{k}$ with $B_{1} / \bar{B}_{1}=$ $\left\{0+\bar{B}_{1}, b_{1}+\bar{B}_{1}, b_{2}+\bar{B}_{1}, \ldots, b_{n}+\bar{B}_{1}\right\}$. For $j \in\{1,2, \ldots, k\}$ we call $B_{j} / \bar{B}_{j}$ the $j$ th column of $H$. Let $L_{0}=\left\{B_{1}, B_{2}, \ldots, \bar{B}_{k}\right\}$ and

$$
\begin{array}{r}
L_{i}=\left\{b_{i}+\bar{B}_{1}, \sigma_{1}\left(b_{i}+\bar{B}_{1}\right), \sigma_{2} \circ \sigma_{1}\left(b_{i}+\bar{B}_{1}\right), \ldots, \sigma_{k-1} \circ \cdots \circ \sigma_{1}\left(b_{i}+\bar{B}_{1}\right)\right\}, \\
i \in\{1,2, \ldots, n\},
\end{array}
$$

and call each $L_{i}$ a line. Further we let $\mathscr{L}=\left\{L_{0}, L_{1}, \ldots, L_{n}\right\}$ and $\mathscr{L}^{*}=$ $\mathscr{L} \backslash\left\{L_{0}\right\}$. For $L \in \mathscr{L}$ and $j \in\{1,2, \ldots, k\}$ let $L^{j}$ denote the coset in $L$ which is in the $j$ th column of $H$. For $0 \neq x \in B_{1} \cup \cdots \cup B_{k}$, say $x \in B_{i}$, denote by $L_{x, i}$ the unique line $L$ such that $x \in \bigcup L$. Further, for $0 \neq x \in$ $B_{1} \cup \cdots \cup B_{k}$ let $C(x)=\left\{i \in\{1,2, \ldots, k\} \mid x \in B_{i}\right\}$ and $P(x)=\{(i, j) \in$ $\left.\{1,2, \ldots, n\} \times\{1,2, \ldots, k\} \mid x \in L_{i}^{j}\right\}$. Thus $C(x)$ denotes the columns of $H$ in which $x$ appears and $P(x)$ gives the coordinates of the cosets which contain $x$ in the array of lines and columns.

For $L_{i}, L_{j} \in \mathscr{L}^{*}$ we write $L_{i} \sim L_{j}$ if there exists $\left\{i_{1}, \ldots, i_{m}\right\} \subseteq\{1,2, \ldots, n\}$ and $x, x_{i_{1}}, \ldots, x_{i_{m-1}}, y \in B_{1} \cup \cdots \cup B_{k}$ such that $x \in \cup L_{i} \cap \cup L_{i_{1}}, x_{i_{1}} \in$ $\bigcup L_{i_{1}} \cap \bigcup L_{i_{2}}, \ldots, x_{i_{m-1}} \in \bigcup L_{i_{m-1}} \cap \bigcup L_{i_{m}}$ and $y \in \bigcup L_{i_{m}} \cap \bigcup L_{j}$. It is straightforward to verify that $\sim$ is an equivalence relation on $\mathscr{L}^{*}$. We call the equivalence classes connected components and say $\mathscr{L}^{*}$ is connected when $\mathscr{L}^{*}$ is a connected component.

When $\mathscr{L}^{*}$ is connected, one can find (after possibly reordering $\mathscr{L}^{*}$ ) a set $\left\{x_{1}, \ldots, x_{m}\right\}$ such that $\left\{l \mid(l, j) \in P\left(x_{i}\right)\right.$ for some $\left.j, 1 \leq j \leq k\right\}=\left\{l \mid l_{i-1} \leq\right.$ $\left.l \leq l_{i}\right\}$ for $i \in\{1,2, \ldots, m\}$, where $l_{0}=1$ and $l_{m}=n$. We call $\left\{x_{1}, \ldots, x_{m}\right\}$ a set of generators. For $l_{i-1} \leq l \leq l_{i}$, let $\left\{j_{1}^{l}, \ldots, j_{k_{l}}^{l}\right\}$ denote the columns $j \in\{1,2, \ldots, k\}$ such that $x_{i} \in L_{l}^{j}$. From this we note that $L_{x_{i}, j_{1}^{l}}=L_{x_{i}, j_{2}^{l}}=$ $\cdots=L_{x_{i}, j_{k}^{\prime}}$.

A sequence $A=\left(a_{1}, a_{2}, \ldots, a_{m}\right)$ where $a_{j} \in \bigcup_{i=1}^{k} B_{i}$ is a good sequence for $x_{i} \in\left\{x_{1}, \ldots, x_{m}\right\}$ if

(a) $C\left(a_{k}\right)=C\left(x_{k}\right), k \in\{1,2, \ldots, m\}$,

(b) $\forall k \in\{1,2, \ldots, m\}, \forall j \in\{1,2, \ldots, k\}, x_{k} \in \bar{B}_{j}$ implies $a_{k} \in \bar{B}_{j}$,

(c) $\exists l, l_{i-1} \leq l \leq l_{i}$ and $p \in\left\{j_{1}^{l}, \ldots, j_{k_{l}}^{l}\right\}$ such that $a_{i} \in \bar{B}_{p}^{*}$,

(d) $\forall k, j \in\{1,2, \ldots, m\}, \forall k_{1} \in C\left(x_{k}\right), \forall k_{2} \in C\left(x_{j}\right)$

$$
L_{x_{k}, k_{1}}=L_{x_{j}, k_{2}} \Rightarrow L_{a_{k}, k_{1}}=L_{a_{j}, k_{2}} \text {. }
$$

From $L_{x_{i}, j_{1}^{l}}=\cdots=L_{x_{i}, j_{k^{\prime}}^{\prime}}$ and part (d) of the definition of good sequence we obtain $L_{a_{i}, j_{1}^{\prime}}=\cdots=L_{a_{i}, j_{k_{l}}}$. We give one further definition, and then we present our main characterization result. 
Let $x_{i} \in\left\{x_{1}, \ldots, x_{m}\right\}$ and let $A=\left(a_{1}, a_{2}, \ldots, a_{m}\right)$ be a good sequence for $x_{i}$. For $x \in G^{*}$ define

$$
A(x)=\bigcap_{\substack{(l, j) \in P(x) \\ 1 \leq l \leq l_{1}}} L_{a_{1, j}}^{j} \cap \cdots \cap \bigcap_{\substack{(l, j) \in P(x) \\ l_{m-1} \leq l \leq l_{m}}} L_{a_{m}, j_{1}^{\prime}}^{j}
$$

THEOREM 2.2. Let $H=B_{1} / \bar{B}_{1}{\underset{\sim}{\sigma_{1}}}_{\sigma^{\prime}} \cdots{\underset{\sim}{\sigma_{k-1}}}_{\sigma_{k}} B_{k} / \bar{B}_{k}$. Then $N=M(G, k, H)$ is a near-field if and only if

(1) $N$ is 0 -symmetric,

(2) $\forall 0 \neq x \in B_{1} \cup \cdots \cup B_{k}, \bigcap_{i \in C(x)} B_{i}=\{0\}$,

(3) $\bigcup_{i=1}^{n} \cup L_{i}=G^{*}$,

(4) $\mathscr{L}^{*}$ is connected with a set of generators $\left\{x_{1}, \ldots, x_{m}\right\}$,

(5) $\forall_{i} \in\{1,2, \ldots, m\}$, for all good sequences $A=\left(a_{1}, \ldots, a_{m}\right)$ for $x_{i}$, $\exists x \in G^{*}, A(x)=\varnothing$ or $\exists j \in\{1,2, \ldots, k\}, \exists x \in B_{j}^{*}, A(x) \cap B_{j}=\varnothing$.

Proof. We first show that the conditions are necessary. If $N$ is not 0 -symmetric then it is known (see [3]) that $N \cong M_{C}\left(Z_{2}\right)$. But this is impossible in our situation since the identity map is in $N$. Suppose now $\bigcap_{i \in C\left(x_{0}\right)} B_{i} \neq\{0\}$ for some $0 \neq x_{0} \in B_{1} \cup \cdots \cup B_{k}$, say $0 \neq b \in \bigcap_{i \in C\left(x_{0}\right)} B_{i}$. Define $f: G \rightarrow G$ by $f\left(x_{0}\right)=x_{0}+b$ and $f(y)=y$ for $y \neq x_{0}$. Then $f \in N$, a contradiction to $N$ being a near-field. If $\bigcup_{i=1}^{n} \cup L_{i} \subsetneq G^{*}$, define $g: G \rightarrow G$ by $g(x)=x, x \in \bigcup_{i=1}^{n} \bigcup L_{i}$ and $g(y)=0$, otherwise. Again, $g \in N$, a contradiction.

If $\mathscr{L}^{*}$ is not connected let $C_{1}$ and $C_{2}$ be distinct connected components. Define $h: G \rightarrow G$ by $h(0)=0, h(x)=0$ for those $x$ such that there exists $L \in C_{1}$ with $x \in \bigcup L$ and $h(y)=y$ otherwise. Once again a contradiction is obtained since $h \in N$.

To show that property (5) is necessary let $i \in\{1,2, \ldots, m\}$ and let $A=$ $\left(a_{1}, \ldots, a_{m}\right)$ be a good sequence for $x_{i}$ such that $A(x) \neq \varnothing$, for each $x \in G^{*}$ and $A(x) \cap \bar{B}_{j} \neq \varnothing$ for all $j \in\{1,2, \ldots, k\}$ and all $x \in B_{j}^{*}$. Define a function $f: G \rightarrow G$ by

$$
\begin{gathered}
f\left(x_{k}\right)=a_{k}, \quad k=1,2, \ldots, m ; \\
f(x)=y_{x} \in A(x) \cap \bar{B}_{j}, \quad x \in B_{j}^{*} \backslash\left\{x_{1}, \ldots, x_{m}\right\}, \quad j=1,2, \ldots, k ; \\
f(0)=0 ; \\
f(x)=y_{x} \in A(x), \quad \text { otherwise. }
\end{gathered}
$$

We first show $f \in N$. Let $l \in\{1,2, \ldots, n\}$, say $l_{i-1} \leq l \leq l_{i}$. Let $y_{1}, y_{2} \in L_{l}$, say $y_{1} \in L_{l}^{i_{1}}, y_{2} \in L_{l}^{i_{2}}$. We must show $L_{f\left(y_{1}\right), i_{1}}=L_{f\left(y_{2}\right), i_{2}}$. However, since $f\left(y_{i}\right) \in A\left(y_{i}\right), i=1,2$, we have $f\left(y_{1}\right) \in L_{a_{i}, j_{1}^{\prime}}^{i_{1}}, f\left(y_{2}\right) \in L_{a_{i}, j_{1}^{j}}^{i_{2}}$ and so $L_{f\left(y_{1}\right), i_{1}}=L_{a_{i}, j_{1}^{l}}=L_{f\left(y_{2}\right), i_{2}}$ as required. From this we obtain $f\left(L_{l}\right) \subseteq L_{f\left(y_{1}\right), i_{1}}$. 
Now, since $f\left(L_{0}\right) \subseteq L_{0}$ by definition, we have $f \in N$. From property (c) of the definition of good sequence there is some line $L_{i} \in \mathscr{L}^{*}$ such that $f\left(L_{i}\right) \subseteq L_{0}$ so $f$ cannot be invertible, contrary to $N$ being a near-field.

For the converse let $f \in N$ and suppose that $f(x)=0$ for some $x \in G^{*}$. We show $f$ must be zero map. Consequently $N$ has no divisors of zero and thus, since a finite near-ring without divisors of zero is a near-field, we have the result.

Let $x \in \bigcup L_{l}$ for some $l$, say $l_{i-1} \leq l \leq l_{i}$. Since $x_{i} \in L_{l}^{j}$ for $j \in$ $\left\{j_{1}^{l}, \ldots, j_{k_{l}}^{l}\right\}, f\left(x_{i}\right) \in B_{j}$. If $f\left(x_{i}\right) \neq 0$, then $f\left(x_{1}\right)=b_{1}, \ldots, f\left(x_{i}\right)=b_{i}, \ldots$, $f\left(x_{m}\right)=b_{m}$ defines a good sequence for $x_{i}$. But in this case we have $f(x) \in A(x)$ for $x \in G^{*}$ and $f(x) \in A(x) \cap \bar{B}_{j}$ for $x \in \bar{B}_{j}^{*}, j \in\{1,2, \ldots, k\}$, contradicting property (5). Thus $f\left(x_{i}\right)=0$. But then $f\left(L_{l}\right) \subseteq L_{0}$ for all $l_{i-1} \leq l \leq l_{i}$. If $1<i<m$ then $x_{i-1} \in L_{l_{i-1}}$ and $x_{i+1} \in L_{l_{i}}$. Again using property (5), by repeating the same argument, we have $f\left(L_{l}\right) \subseteq L_{0}$ for all $l, l_{i-2} \leq l \leq l_{i+1}$. Continuing in this manner we obtain $f\left(L_{l}\right) \subseteq L_{0}$ for all $l \in\{1,2, \ldots, n\}$. But then $f(x) \in \bigcap_{i \in C(x)} B_{i}$ for all $x \in G^{*}$. From property (2), $f=0$.

In the "without quotients" situation, that is, when $\bar{B}_{j}=\{0\}$ for all $j \in$ $\{1, \ldots, k\}$, properties (2) and (5) are automatically fulfilled and here we have $\bigcap_{l=1}^{n} \cup L_{l}=\left(\bigcup_{j=1}^{k} B_{j}\right)$. Thus we have the following.

Corollary 2.3. Let $H=B_{1} /\{0\} \underset{\sim}{\underset{\sigma_{1}}{x}} \cdots{\underset{\sim}{\sigma_{k-1}}}_{B_{k}} B_{\{0\}}$. Then $N=$ $M(G, k, H)$ is a near-field if and only if

(1) $N$ is 0 -symmetric,

(2) $\bigcup_{j=1}^{k} B_{j}=G$,

(3) $\mathscr{L}^{*}$ is connected.

We conclude the paper with an example which shows that the conditions of the above theorem need not hold. This meromorphic product fulfills (1)-(4) but not (5) and therefore determines a near-ring which is not a near-field.

EXAMPLe 2.4. Let $G=\left(Z_{2}\right)^{4}$ with the usual basis $\left\{e_{1}, e_{2}, e_{3}, e_{4}\right\}$. Let $B_{1}=G, B_{1}=\left\langle e_{1}+e_{2}, e_{3}+e_{4}\right\rangle, B_{2}=G, B_{2}=\left\langle e_{1}, e_{2}+e_{4}\right\rangle, B_{3}=\left\langle e_{1}, e_{2}, e_{4}\right\rangle$, $B_{3}=\left\langle e_{1}\right\rangle, B_{4}=\left\langle e_{1}, e_{3}, e_{2}+e_{4}\right\rangle, B_{4}=\left\langle e_{1}+e_{2}+e_{3}+e_{4}\right\rangle, B_{5}=\left\langle e_{1}, e_{3}+e_{4}\right\rangle$, $B_{5}=\{0\}, B_{6}=\left\langle e_{1}, e_{2}+e_{3}\right\rangle$ and $B_{6}=\{0\}$. The following scheme determines a meromorphic product:

$$
\begin{gathered}
e_{1}+B_{1} \mapsto e_{1}+e_{2}+B_{2} \mapsto e_{2}+\bar{B}_{3} \mapsto e_{1}+\bar{B}_{4} \mapsto e_{3}+e_{4}+\bar{B}_{5} \mapsto e_{1}+\bar{B}_{6}, \\
e_{1}+e_{4}+\bar{B}_{1} \mapsto e_{4}+B_{2} \mapsto e_{4}+\bar{B}_{3} \mapsto e_{1}+e_{3}+\bar{B}_{4} \\
\mapsto e_{1}+e_{3}+e_{4}+\bar{B}_{5} \mapsto e_{2}+e_{3}+\bar{B}_{6} .
\end{gathered}
$$


Using $x_{1}=e_{1}+e_{3}+e_{4}, x_{2}=e_{4}$ and $A=\left(e_{3}+e_{4}, e_{1}+e_{2}\right)$ as a good sequence for $x_{1}$, one defines a function in $M(G, 6, H)$ which is not invertible.

\section{References}

[1] P. Fuchs and C. J. Maxson, 'Near-fields associated with invariant linear $k$-relations', Proc. Amer. Math. Soc. 103 (1988), 729-736.

[2] J. D. P. Meldrum, Near-rings and their links with groups (Research Notes in Math. 134, Pitman, London, 1986).

[3] G. F. Pilz, Near-rings (2nd ed., North-Holland, Amsterdam, 1983).

[4] R. Remak, 'Über die Darstellung der endlichen Gruppen als Untergruppen direkter Produkte', J. Reine Angew. Math. 163 (1930), 1-44.

[5] R. Remak, 'Über Untergruppen direkter Produkte von drei Faktoren', J. Reine Angew. Math. 166 (1932), 65-100.

[6] H. Wielandt, Permutation groups through invariant relations and invariant functions (Lecture notes, Ohio State University, Columbus, 1969).

\section{Department of Mathematics \\ Texas A \& M University \\ College Station, Texas 77843 \\ U.S.A.}

